\title{
Towards Product Customization and Personalization in IoT-enabled Cloud Manufacturing
}

\author{
Chen Yang ${ }^{1,2}$, Shulin Lan ${ }^{1}$, Weiming Shen ${ }^{2}$, George Q. Huang ${ }^{1}$, Xianbin Wang ${ }^{2}$, Tingyu $\operatorname{Lin}^{3}$ \\ ${ }^{1}$ HKU-ZIRI Lab for Physical Internet, Dept. of Industrial and Manufacturing Systems Engineering, \\ The University of Hong Kong, Pokfulam Road, Hong Kong, P.R. China \\ ${ }^{2}$ Dept. of Electrical and Computer Engineering, Western University, London, ON, Canada \\ ${ }^{3}$ State Key Lab of Intelligent Manufacturing System Technology, \\ Beijing Simulation Center, Beijing, P.R. China \\ \{wzhyoung@163.com, lanshulin1349@sina.com,wshen@ieee.org, \\ gqhuang@hku.hk, xianbin.wang@uwo.ca, lintingyu2003@sina.com\}
}

\begin{abstract}
Customized/personalized products are gaining more shares in today's product market. Such products need collective efforts from consumers, manufacturers and third parties. This requirement has not been well addressed due to both market and technology factors. On the other hand, the Internet of Things (IoT) provides real-time sensing/actuating ability and fast transmission capability of data/information, so that remote operation of manufacturing activities and efficient collaboration among stakeholders are greatly facilitated. This provides great opportunities to address the requirement mentioned above. Thus we propose a full-connection model of product design and manufacturing in the IoT-enabled cloud manufacturing environment. The proposed model uses social networks to connect multiple parties and facilitate open innovations, and use IoT to glue physical space to cyber space and cloud manufacturing to provide various elastic services, so that the on-demand workspace, interaction, information sharing or collective problem solving are enabled. We also propose a supporting infrastructure for this model using the latest information and communication technologies. Finally, we present a case study of an RFID enabled production system for customized and personalized product with the ability to enable a
\end{abstract}


new paradigm of "dynamic processes and close collaborations among different roles" and ensure robust production.

\section{Keywords}

Cloud Manufacturing; Internet of Things; Concurrent Engineering; Social Network; Big Data; RFID (Radio frequency identification)

\section{Introduction}

With the increasingly developed industry and richer material things available, a popular trend is the customized and personalized product (CPP), which can best meet individual customers' needs. The paradigm of CPP is imperative for many companies to survive in the fragmented, diversified, and competitive marketplace [1]. However, to provide CPP, the manufacturers need more diverse (and more) resources and capabilities, which would cost them too much both on facility construction and skilled recruits. Moreover, the utility rate of those resources and capabilities can fluctuate greatly at different time, leading to much waste. Thus how to provide CPP at low cost is a noteworthy problem and needs to be solved.

Cloud manufacturing (CMfg) which can provide mass manufacturing resources and capabilities as services via the Internet [2] offers a promising solution. It successfully achieves the aggregation of open manufacturing resources and capabilities that are distributed worldwide and are "sufficiently" large from the customer point of view. CMfg achieves the pervasive presence of various CMfg services around users, who can acquire and release services on actual demand and pay as "they" go. Then the effective utilization of those services will largely depend on manpower, who uses them to conduct innovative activities of designing and manufacturing CPPs. 
From the perspective of competences, inter-company collaboration and crowd innovation will solicit great contributions to the design and manufacturing of CPPs, while most current innovations are achieved inside companies [3]. At least, customers will closely work with the related parties in the product design and manufacturing stages to well express their needs. With the advent of social networks (SN), open innovations are elicited and greatly facilitated. Several conceptual frameworks [4][5][6][7] are proposed on the exploitation of $\mathrm{SN}$ to develop the manufacturing sector. IoT, which can provide real-time information about manufacturing things and use actuators to control them, can help CMfg reinforce its ability on closing the loop of sensing, decision-making and actuation and thus greatly improve collaboration efficiency and experience. However, little concrete effort has yet been made to leverage IoT-enabled CMfg and SN for the integrated design, supply chain management, production, marketing and sales and aftersales of CPP.

Thus, this paper tries to propose a solution to embrace human resources outside the organization borders in the CPP design and manufacturing stages dynamically on demand. The paper is organized as follows: Section 2 reviews the related research literature; Section 3 proposes a new model of full CPP lifecycle; Section 4 describes a supporting framework for design and manufacturing of CPP; Section 5 provides an RFID (Radio frequency identification) enabled case study with new engineering processes; and Section 6 presents some discussions and Section 7 provides brief concluding remarks.

\section{Related Work}

The development of CMfg provides a natural eco-system for the open CPP design and manufacturing for three reasons. First, CMfg can well support SNs (e.g., Facebook) which have highly dynamic and unpredictable demands for storage and computing 
capabilities. Second, CMfg which can deliver on-demand services ("XaaS”[8], such as 3D printing service) via the management of various soft and hard manufacturing resources and capabilities can also accommodate huge amounts of data from the Internet, enterprise information systems, and IoT systems. When mass service providers and consumers enter the CMfg eco-system, then this will further attract more resources and users, forming a virtuous cycle. Then, the available CMfg services will be greatly enriched, benefiting all the participant parties. Third, when large amounts of participants exist in SN and the CMfg environments, they have more chances to form dynamic communities with common interests, which can collectively contribute to the better development of CPP. As indicated, CMfg can provide the best-fit virtual workspace dynamically on demand for such communities, which can facilitate timely collaboration.

There are some literature on IoT-enabled Cloud Manufacturing. Li et al [2] proposed the concept of CMfg which merges IoT's ability to pervasively connect manufacturing things. Tao et al. [25] further explored the potentials of merging IoT (with intelligent perception and connection ability) into CMfg from aspects of architecture, key technologies and challenges. Bi et al. [16] investigates the impact of IoT on enterprise manufacturing systems (EMSs) by analyzing the evolution of EMSs and IT infrastructure, and the new advantages brought by IoT. Liu et al. [26] compared industry 4.0 featuring the employment of IoT with CMfg. However, research on how the IoT and CMfg can better be utilized for customized and personalized products needs to be substantially conducted, which also involves effective organization of talents across borders of organizations or areas.

Some work has been done on the application of SN in manufacturing, but mostly at the conceptual level. Tao et al. [6] reviewed the evolution of advanced manufacturing systems and concluded the socialization trend, which is showcased in the resource 
sharing, the value creation and the user participation. Jiang et al. [5] proposed the concept of social manufacturing and analyzed its three core aspects from the configuration, operation and management perspectives. Wu et al. [4] formulated a SN analysis approach to analyze the overall collaborative structure, key actors and collaboration communities of the socio-technical network generated by cloud-based design and manufacturing systems. Jeong et al. [18] proposed an evaluation-committee recommendation system for national $\mathrm{R} \& \mathrm{D}$ projects via online detection of researcher connections on social networks. However, these efforts either have no concrete approaches or do not address the issue of new models and processes enabled by CMfg, IoT and $\mathrm{SN}$ for CPP.

Traditional methods focus on the design for manufacture and assembly, attempting to consider all the factors and constraints of product lifecycle (via collaboration with related stakeholders from later stages) in the design stage. The benefits, such as reduced iterations and manufacture errors, are obvious [9]. However, it is very difficult, if not impossible, to do so at first. Many problems cannot be uncovered and predicted until specific activities start. Thus even though concurrent engineering practice is taken, the collaborations with design resources and capabilities are needed at the later stages. In other words, during any stage, it is possible that collaborations with resources and capabilities from other stages will bring benefits to enhance concurrent engineering practice.

Industry 4.0 holds the promise of enabling last-minute changes to production and delivering the ability to respond flexibly to disruptions and failures [10]. This will facilitate the better response to changing needs of designers, manufacturer, consumers, etc. for CPPs. However, most current production methods or systems cannot provide such advanced supports. Zhong et al. [11] proposed an RFID-enabled real-time 
manufacturing execution system for mass-customization production, however, it cannot support the dynamic change of manufacturing processes of CPP after the CPP design has been put into production, e.g., when some revisions are made afterwards. On the other hand, the dynamic configuration of CPPs closely relates to the consumers whose involvement is essential and facilitated by the latest technologies, such as CMfg, SN and IoT. However, there still lacks a well-fitted framework that can exploit the advantages of these latest technologies to achieve the ideal promised by the open product design and manufacturing.

\section{Lifecycle Model for Customized and Personalized Product}

The pervasive sensing and advanced connectivity between IoT devices pushes the collaboration in the product lifecycle to a new level with high visibility, efficiency and flexibility. Sudarsan et al. [9] proposed a PLM (product lifecycle management) epicycle but with limited interactions, for example, no direct feedback from products in use to the design. The direct interactions can effectively reduce the time consumed to get useful information and prevent the potential loss due to the lack of timely information. Thus we propose a full-connection model of $\mathrm{SN}$-based open collaborations in the product lifecycle (Fig. 1), which is equipped with new ICTs, such as IoT, Cloud, SN, and conceives real-time interactions on demand between any two stages to facilitate any kind of collaborative problem solving and product personalization. 


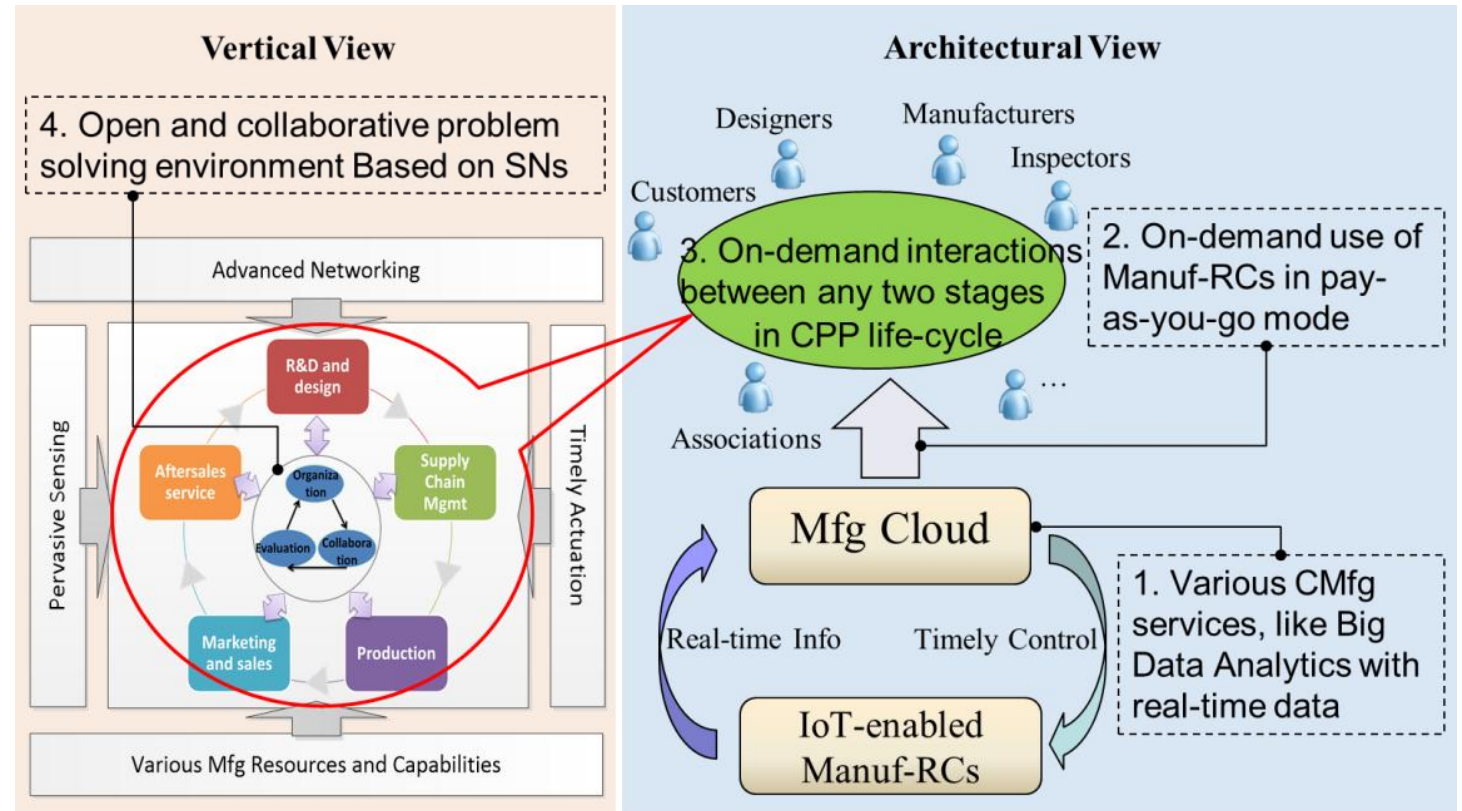

Figure 1. CMfg enabled open collaboration model

We adopt the classification stated in [3], where the full product lifecycle consists of five stages: R\&D and design, supply chain management, production, marketing and sales and aftersales service. Activities in different stages can be conducted concurrently following the concurrent engineering practice.

\subsection{Advantages of the Proposed Model}

The proposed model has the following advantages: first, traditional circular interactions are enhanced with cross-stage interactions to facilitate timely and efficient information and resource sharing, i.e., the on-demand interaction based on the full-connection structure (not only in design-for-X stage) is enabled. Second, IoT-enabled open manufacturing resources and capabilities (dynamic, scalable and seemingly infinite) can be accessed dynamically in short term and in the pay-as-you-go economical manner. Users are allowed to leverage the on-demand growing and shrinking of resources and capabilities. A combination of CMfg and SN can greatly achieve this vision. Third, IoT, big data analytics, and virtual/augmented reality \& 
simulation can also help the collaboration efficiency and experience, which will be justified in Section 4.1.

For example, intelligent prognostics for health monitoring of in-use high-value products during the aftersales service stage through the adoption of IoT can feedback useful usage information to the $R \& D$ and design stage to promote the design of next-generation products, and can pass demands for new parts (predictive maintenance) to supply-chain management and production stages to enable timely part replacement. Through this hyper interaction structure, useful information, or even resources can be easily accessed anywhere they are needed in a real-time manner.

\subsection{Open Problem Solving Environment}

The problem solving process in the product lifecycle includes three steps. The first step is to (dynamically) form communities which involve the potential contributors, according to demands. Then collaborative efforts are made using various media, such as text, video [12], voice, and simulator. Big Data, virtual/augmented reality and simulation and cloud can offer solid help in the full CPP lifecycle. After/during the collaboration, evaluation is a must to guarantee user satisfaction and quality of results. The unsatisfactory results may give rise to a reorganization of communities, for example to include more talents, or iteration of problem solving process inside the original communities. Those three kinds of activities form a close loop to facilitate effective and efficient collaborations in the CPP's lifecycle.

The crowd possibly involved in communities has talent to make important contributions. A famous example is "The DARPA Robotics Challenge" [24], which attracts some of the most advanced robotics research and development organizations in the world to compete a series of challenge tasks. Different designs will collectively benefit the development of humanoid robotics from "Boston Dynamics". Domain 
experts can be invited to join the communities for the effective problem solving. Open calls can reach vast people through $\mathrm{SN}$, which is prevalent and easily accessible using smart phones. Due to the massive user base, through collaborations (e.g. brainstorm), creative ideas and techniques can be acquired in a high possibility. SN which is pervasive among the vast majority provides an ideal platform to tap into the competences of vast human resources outside of the organizations for better CPP.

To guarantee right and efficient collaboration, flexible information-and-resource sharing methods (due to the continuously changing demand for sharing) considering the security and privacy factors should be established. They can be methods of subscription (event) based information forwarding, (dynamic) community based direct discussion, etc. CMfg and SN can effectively support those methods (e.g., subscription based open calls on Facebook).

\section{Product Customization and Personalization in IoT-Enabled Cloud}

\section{Manufacturing}

\subsection{System Architecture}

Fig. 2 illustrates the main components of the system architecture: five different roles of users involved and the supporting infrastructure.

\subsubsection{Five Roles of Users Involved}

Five kinds of stakeholders are involved in the open CPP design and manufacturing. Customer refers to individuals or companies who need the CPPs. Dynamic communities and professional associations are third parties who have common interests or own the know-how concerning the CPPs. Product designer is the main contributor of CPP design, while dynamic communities \& professional associations and customer will also be involved for better CPP design with good user experience and creative solutions. Manufacturer and Quality inspector are responsible for the processing/assembling of 
materials/parts and quality assurance/testing respectively. In different stages of the CPP lifecycle, collective efforts from the five kinds of people are necessary for better products and lower cost and time.
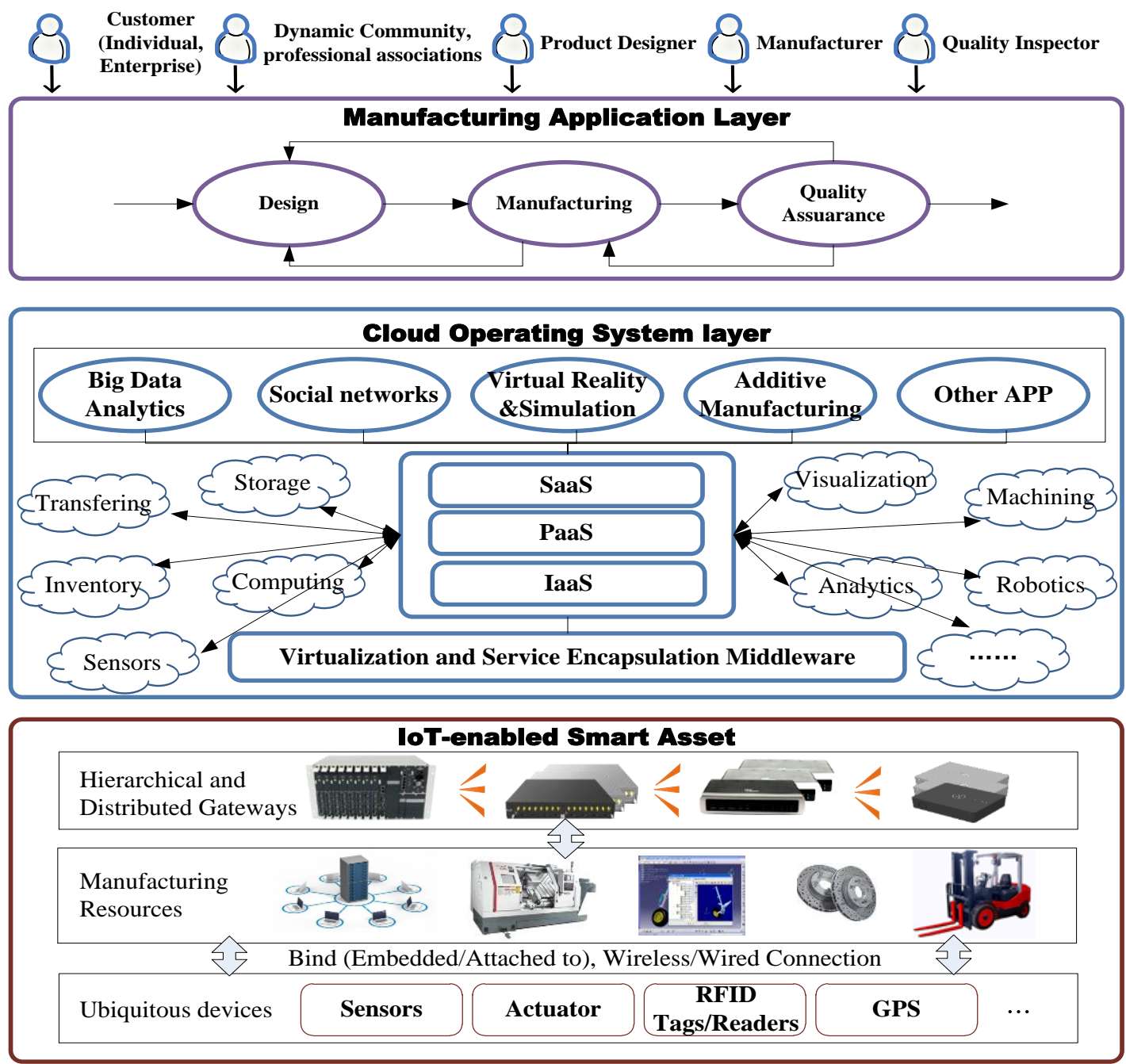

Figure 2. Supporting Architecture of IoT-enabled CMfg for CPPs

\subsubsection{Supporting Infrastructure}

The supporting infrastructure consists of three layers: IoT-enabled smart asset, cloud operating system and manufacturing application.

\section{1) IoT-enabled Smart Asset Layer}

Manufacturing resources including soft resources (e.g., design models and software) and digitalized hard resources (e.g., CNC) are in the position to be monitored and controlled remotely. For traditional hardware facility, the embedding or attaching of tiny 
electronics, such as sensors, RFID tags and actuators can interface them with the resulting cyberspace to provide status information and receive and execute the control orders. There are also autonomous objects that inherently contain sensing, decision-making and actuating modules like intelligent agents [19]. All manufacturing resources are networked and exchange information with each other using the wireless/ wired communication approaches. Hierarchical and Distributed Gateways are designed to facilitate coordination and interoperation among different levels of assets via establishing hybrid management network. The gateways can support freely create upward and downward hierarchy to realize multi-dimensional cooperation. With built-in data analytic algorithms and communication rules, smart gateways are capable of executing and assigning tasks collaboratively. The whole manufacturing IoT evolves under the hybrid control paradigm of centralized governance and distributed autonomy.

\section{2) Cloud Operating System Layer}

The cloud operating system layer provides the efficient management of various manufacturing resources, which can be widely accessed in the form of CMfg services via the Internet. First and foremost, massive amounts of smart asset are virtualized into a resource pool and encapsulated as services to enable the on-demand delivery of services. Virtualization makes it possible to dynamically divide resources into virtual units or flexibly combine them (with other resources) to a logic unit to meet diversified demands, while the functions of those logic units can be encapsulated using SOA paradigm to serve geo-distributed users. The basic services provided by the smart objects can be orchestrated to be more complicated services to offer various competences [13].

Like in cloud computing, the cloud operating system could use three delivery models (IaaS, Paas, and SaaS) to provide services, which can be typical cloud computing 
services like storage, computing, analytics and visualization [14] [15], and CMfg-specific services like machining, inventory, robotics, transferring, sensing, etc.

With unprecedented capabilities, the CMfg cloud lays a solid foundation for various powerful technologies and their supported services. We here list a few highly-related ones that can contribute to our work. Due to the advances in sensor technology and pervasive devices, big data analytics will play a vital role in manufacturing with overwhelming data collected at an unprecedented scale and speed. It helps to analyze heterogeneous big data acquired from many sources like sensors, SN and internet, extract knowledge in a timely manner and facilitate faster and better decision-making in various manufacturing applications.

SN offers like-minded individuals or professional associations a public space for communication and sharing and thus presents opportunities to tap into the competencies of people beyond the organization boundaries. $\mathrm{SN}$ can also deliver the latest updates to users who subscribe the concerned information from their friends, colleagues or interested communities. In traditional online communities, users have to browse other people's homepages or blogs one by one to acquire updates, which may waste time when there are no (related) updates. With rich means to communicate and share contents, like live video broadcasting, photograph or short videos, participants can get a full understanding on what like-minded people are doing and start effective instant communications.

Virtual/Augmented reality and simulation can replicate a virtual environment that simulates a physical presence in places in the real world or an imagined world, allowing the user to interact in that world. With increasingly widespread sensors and actuators (and powerful connections between them), more parameters can be monitored continuously in a real-time manner. Those real data can be used to create and drive more 
vivid virtual/augmented reality and simulation. This powerful paradigm will push many aspects of manufacturing, such as virtual product developing, to a higher level. A concrete example is virtual assembly after the CPPs are designed or during the dynamic production processes. The training of new operators can also be realized in an immersive and vivid manner. With augmented reality technologies, engineers can manipulate physical machines in a virtual environment anywhere and anytime, while the potential hazards in the real environment can be avoid. Holographic Laser Projection is noteworthy. With IoT, virtual/augmented reality and simulation can be greatly enhanced by real-time data driven approaches based on the practical data from the physical systems. Operations can be conveyed to actuate the real systems.

\section{3) Manufacturing Application Layer}

To make the presentation succinct, we here only consider three main stages or activities in the CPP lifecycle. As shown in Fig. 2, they are design, manufacturing and quality assurance. Intense collaborations among different parties from a stage or across stages are needed to improve the overall quality, lead-time, cost, etc. The manufacturing cloud is an ideal platform for such collaborations when all the data can be conveniently stored, flexibly shared and efficiently handled with various elastic capabilities powered by the cloud.

In the open CPP design and manufacturing paradigm, individual requirements can be posted to $\mathrm{SN}$ where friends, individual designers, design companies, professional associations or any other related people can jointly engage in the design of conceptual models for CPPs. Such brainstorming in a reasonably large scale can greatly contribute to the generation of ideal concepts for the product design, due to the diverse background, life experience, thinking mode, competence, etc. In this process, customers can fully communicate with the designers and other involved people. Manufacturers and 
inspectors may also involve in this stage to impose constraints from production, assembly and inspection perspectives. Also at the same time they can start part of production work and inspection plan following the concurrent engineering practice.

Then the approved product design will be put into the production. During the production stage, real-time production data and parameter data about the product can be collected and feedback to customers, production managers and inspectors to review. Virtual/Augmented reality \& simulation can be employed to try the personal product to be completed and inspect its quality. The close loop for real-time information flow between design, production and inspection enables last-minute changes to production, where users can conveniently change the design of products to better meet their personal demands [20], or/and inspectors can feedback the potential problem to production manager to change the processes (or the remanufacturing of parts). Even inside the production stage, different processing centers can share the disturbances or important events (e.g., the finishing of products) in a real-time manner (enabled by IoT technology) to make better decisions adaptively.

To insure the quality of CPPs, inspection is necessary and performed when the products have been manufactured or are being manufactured. Sensors can be mounted on the parts/semi-products or deployed on the machine tools as needed to detect the quality parameters. Related data from sensors and machines can be correlated and analyzed to promote quality assurance and control. When data analytics indicate that the performance of machine facilities severely degrades, preventive maintenance can be conducted before the machine breakdowns practically occur to reduce the potential loss (by ensuring the first-time correctness of accomplished products [16]). 


\subsection{Unique Characteristics}

To realize the paradigm of CPPs, product design and manufacturing processes are usually conducted in an open, parallel, distributed and collaborative manner. The proposed architecture in Section 4.1 can lend strong support to this. Comparing to the models of CMfg overviewed in [27] and [28], our proposed model has been consolidated with IoT's ability of real-time sensing and actuation of objects, and social-network's connection of open social talents. Specifically, at the bottom layer, the hierarchical and distributed smart gateways that will extend to the manufacturing cloud are employed to facilitate coordination and interoperation among different levels of assets via establishing hybrid management network, and realize operation synchronization through handling manufacturing data collected from assets with high frequency. With built-in data analytic algorithms and communication rules, smart gateways are capable of executing and assigning tasks collaboratively. At the upper layer, based on IoT's real-time information and big data analytics, social network can tap into open human resources and capabilities and involve customers to further facilitate the product customization and personalization.

The supported unique characteristics in the dimension of collaborations for CPPs are shown as the following:

\section{1) Inter-organization}

Fierce competition and sophisticated products necessitate loose or tight collaborations between organizations which have complementary competences or resources. More manufacturers will embrace the open innovation paradigm which involves the collaboration across the borders of organizations. The organizations and the vast majority can jointly contribute to a better solution for CPPs on design, production and assembly aspects. 


\section{2) Cross-stage}

Traditional collaboration mostly occurs between two consecutive stages in the full product lifecycle. With increased connectivity and visibility, cross-stage information sharing and interactions are facilitated and can significantly increase productivity and product quality. Traditional design for manufacture and assemble in concurrent engineering is not enough for sophisticated products, as stated in Sect. II. Consequently, cross-stage interactions are needed to ensure the effective involvement of the necessary resources and people in any other stages. This means a lot of parallel and iterative processes, which will improve the product quality and reduce the cost and time.

\section{3) Multi-discipline (industry)}

Sophisticated products usually involve many sub-systems from different disciplines (industries), thus the design and manufacture of those products involve multi-disciplinary efforts. For example, the airplane consists of mechanical, electrical, electronic, aerodynamic, etc. subsystems. Those sub-systems together provide complicated functions to consumers. The design and manufacturing of such products need collective efforts from multiple individuals or organizations that own the know-hows in multiple disciplines/industries.

\section{4) Geo-distributed}

The task of CPPs is usually divided into many subtasks which are undertaken by geo-distributed individuals or organizations in the era of global manufacturing. The parts of Boeing airplanes usually come from the OEMs worldwide, due to the cost considerations. Besides the cost factor, their different strengthens and policies also accounts for such geo-distribution pattern. Just-in-time logistics play an important role while the information sharing can be fast achieved using the internet.

\section{5) Cross-level}


The task of CPPs needs the resources and capabilities with various granularity sizes. They are organized in a hierarchical way, thus cross-level collaboration may be needed to ensure the efficiency [21]. Different layers have varying details which are normally hidden from and may be useful in the upper layers for some manufacturing applications.

\section{RFID-Enabled Customized/Personalized Production Based on}

\section{Multi-agents}

Industry 4.0 characterizes the "last-minute changes to production" [10], which can benefit the open product design and manufacturing of CPPs. We here propose an RFID based method to enable this important feature in the manufacturing cloud.

\subsection{Deployment View of RFID based Production Workshop}

As shown in Fig. 3, the deployment of a RFID-enabled workshop for CPPs consists of five elements: manufacturing cloud, central node (workshop gateway), local wired/wireless network, shop-floor workstations and parts/materials. Other components except the remote manufacturing cloud are deployed in the local workshop. The local wired/wireless network connects the central node and workstations. RFID tags are mounted on (key) parts/materials, while RFID readers are placed in the workstations to read the identity and other information from the tags. This can not only facilitate the capturing and collection of real-time field data (tracing and tracking manufacturing objects, visibility of shop floor) [29], but also provide the precious opportunities to process the parts/materials individually. The network can self-organize if advanced network technologies (e.g., Software-defined Networking and Network Function Virtualization) are used or it consists of self-adaptive parts like WSNs [23]. For example, if workstation 1 cannot connect workstation 3 directly, it will adaptively try the relay method through workstation 2, as shown in Fig. 3. This will greatly enhance the 
robustness of the system to counteract the possible failures and disturbances (e.g., to support the plug and play of new machines).

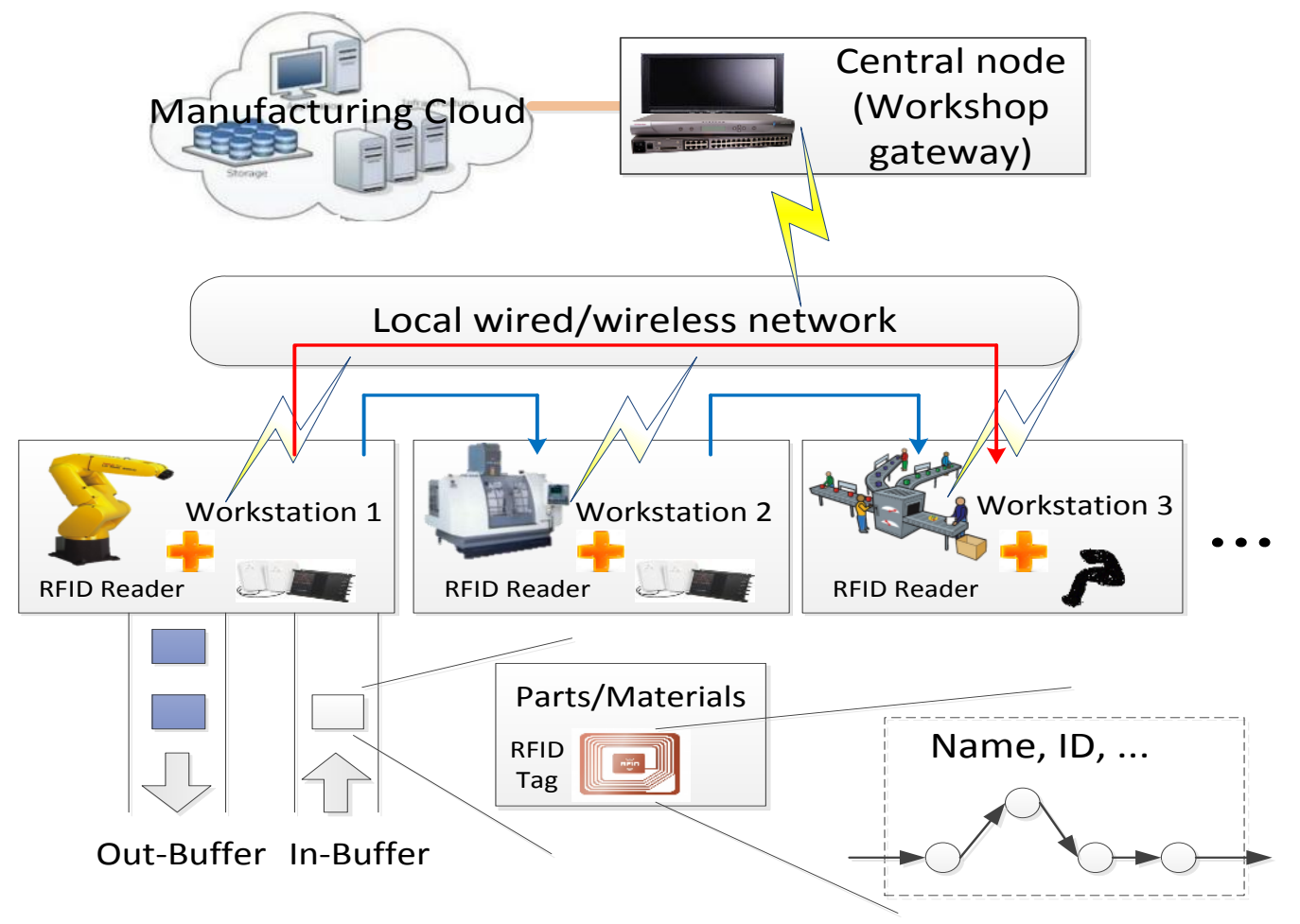

Figure 3. Deployment of RFID-enabled workshop for CPPs

\subsection{RFID-enabled Dynamic Change of Product Design and}

\section{Manufacturing}

In the production stage, when users have some new ideas (e.g., due to incremental learning) or there are some problems (e.g., parts/product quality), collective efforts like discussions are needed with the help of IoT infrastructure, Big Data analytics, SN, virtual/augmented reality \& simulation, and other manufacturing resources/capabilities in the manufacturing cloud. Through the real-time acquisition, analyses and visualization of parts status, designers, manufacturer and quality inspectors can give out new solutions for the problem cooperatively on the SN platforms, mainly the designers improving the CPP design, the manufacturer re-planning the processes and approving the reconfiguration of original designed processes, and the inspectors continuously 
tracking the quality. Such solution is then forwarded to the central node of the workshop where the CPP production is conducted. The processes that have been finished could not been reversed, so the solution can only enable the change of processes yet to be started. Here we will not consider the monetary side (business model for the dynamic change) which needs to be explored in future.

There may be scenarios when the dynamic configuration of production is disabled (e.g., the central node breaks down). In this case, contracts should regulate the management of such situations, or the whole system should stop immediately, which may bring other loss, for example, some processing normally could not stop until it is finished. Special measures are needed to prevent such loss. We do not discuss those issues here.

The central node always stores the latest information on the production configuration, which the workstations need to execute the processing or assembly of parts. The engineering process of RFID-enabled dynamic change of production includes the following steps:

1) All parties achieve consensus on the production change using the $\mathrm{SN}$ in the manufacturing cloud and forward the plan to the central node of the workshop where the production will be conducted. 


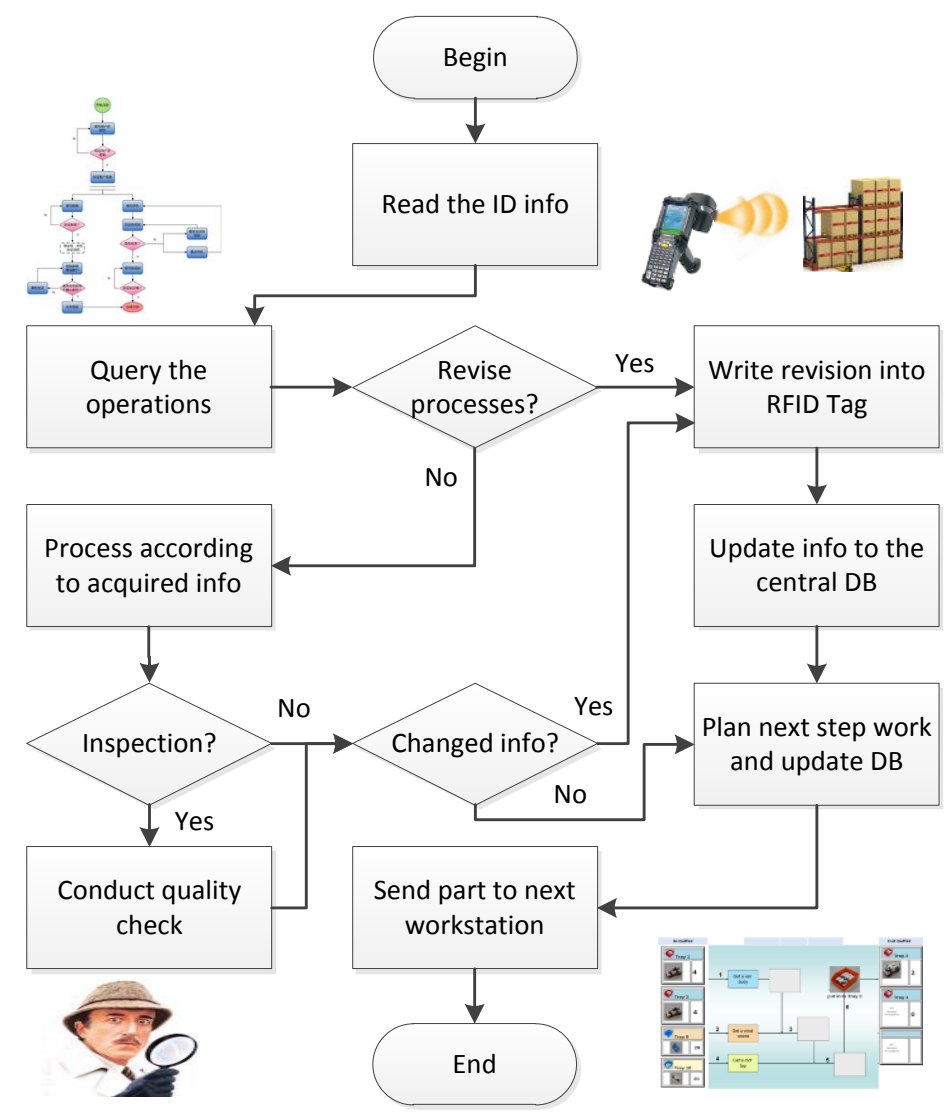

Figure 4. Dynamic service selection process

On every workstation (agent):

2) When a part enters a workstation for processing or assembly, the RFID reader will first read the ID information from the tag on the part and use it to query the latest updates from the central node.

3) The workstation judges whether original production processes have been changed, after it gets the response. If not, then the part can be processed at the current workstation, according to the original plan. Then go to Step 5).

4) If the change of processes exists, the reader needs to write it in the RFID tags and notify the central node about this. Then go to Step 8).

5) After/during the processing on the part, the workstation will need to check whether the inspection should be conducted. If not, go to Step 8).

6) Quality inspectors conduct the inspection. 
7) The workstation will request to get whether there are any updates on the configuration of the part production. If so, go to 4).

8) The workstation will request the routing plan from the central nodes and then send the part to the planned next workstation.

We design Step 7 (a second round of update checking) to avoid the unnecessary routing because the change of original processing/assembling plan may lead to change of the planned next workstation.

\subsection{Robust and Adaptive Manufacturing Systems}

We also design the system with adaptive mechanisms to enhance its robustness and at the same time not to compromise the productivity. The system operates mostly in the centralized manner to be productive and in the distributed paradigm to continue the production when critical failures occur. When the failures are eliminated, the system can be evolved back to work in the centralized way again.

To improve robustness, the communication network should first be reliable and then even if it breaks down at some spots or some workstations fail, the system should also be able to continue the work.

First, self-organized communication networks are adopted to guarantee the reliable interactions between the components of the workshop. As simply indicated in Fig. 3, even some paths fail, the interactions can also be guaranteed by other indirect paths.

Second, the central node should be able to deal with the failures of some workstations and the addition of new workstations. In other words, it should have the ability to make optimal decisions when the available resources change.

Third, the workstations should have the ability to work cooperatively, even when the central node and all the paths to the central node breaks down. In such case, the workstations should know some information about other workstations in advance, at 
least, for example their different skills. The skills mean the work they can perform on the parts/materials. To be efficient, we do not intend to let them negotiate in totally distributed way to get such information. Instead, such information is normally maintained by the central node. The workstations are divided into several lists according to their skills on manufacture or assembly. Changes of those lists will be updated to each workstation during the off-work time or the under-loaded period. The locally stored skill lists in the workstations, the processes information in RFID tags, and the available communication paths enables the cooperative operation of workstations (may be in several groups) with the necessary knowledge to guide the routing of parts/materials, even when the central node or all the communication paths/channels to central node fail.

The switching mechanisms are designed as the following. To join the system, a new workstation will:

1) Initiate the negotiation and build the communication links with its neighbors

2 ) Find the shortest path to the central node

3 ) Report the skills and Get an ID when join the network (the central node will broadcast new update of lists to all workstations)

4) Download the info of other workstations (including skill lists)

5 ) Notify the central node that the workstation is ready for work

6) Do the processing/assembling of parts/materials, as shown in Fig. 4

When a workstation fails to interact with the central node, it means either the central node or all the paths to it break down, as messages between the central node and workstations are routed adaptively. In this case, to continue the work, the workstations have to collaboratively route parts/material to the right workstations according to processes carried in the tags. To enable the smooth transition to the distributed mode, we design the workstation which fails to interact with the central node has to: 
7) Notify other workstations using the current network to change their operating mode

8) Read the next step work of the current part from the RFID tag

9) Request state info of the workstations having required skills (for the work) according to the locally stored skill lists

10) Make optimal decisions on the next destination (workstation) of the part

11 Send the part to the planned workstation

If the central node fails, the whole workstations will work collaboratively in a group. If some network links break down, which may divide the workstation into different connected groups, the workstations will work cooperatively in different groups. When the central node or the network recovers from failures, the central node will notify all the workstations to work in the centralized mode, which is superior to the distributed mode on productivity. The method to achieve this is shown as the following:

12) The central node sends messages to all workstations for the start of the transition

13) Once a workstation receives the message, it will build the relationship with the central node and then act as a slave node after the completion of Step 1)-5)

14) When all the workstations gradually join the centralized network, the system returns to work in the centralized mode again.

To support the gradual return of the workstations, the central node needs the ability of making optimal decisions based on the dynamic available resources.

\subsection{Configurable Optimization Objectives}

When a workstation loses connections to the central node, it can relay its request to the central node via other workstations. When the central node or all the paths to the 
central node breaks down, the workstations can negotiate and collaborate to continue the production, switching into the distributed scheduling mode.

A central problem is the formulation of scheduling problems and the design of optimization algorithms to achieve production efficiency, energy and material saving, emission reduction, more value addition, etc. The robustness and flexibility (plug and play) of the manufacturing system should also be considered.

The length of workstation queues is considered to generate the production schedules. Other factors include the operation speed, disturbances and the health information of workstation, sensed by the IoT facilities and shared through central node or cloud. IoT can be of great help in providing the raw data. Complex event processing (CEP) [22] which tracks and analyzes streams of data from multiple sources is a promising method to infer events or patterns that suggest more complicated circumstances.

\section{Implementation and Discussions}

\subsection{Implementation}

According to the architecture proposed in Section 4.1, we have implemented an IoT-enabled manufacturing cloud in a conglomerate, which integrated 1) hard resources: 1.1) over 300 terabyte (TB) of the Big Data storage, 1.2) hundreds of teraflops per second of the high performance clusters, 1.3) high-speed optical networks, 1.4) hundreds of high-end CNC machining equipment or cellular manufacturing systems from several workshops; 2) soft resources: 2.1) more than 300 sets of tool software for the design and analysis (in mechanical, electronic, control, etc. areas), 2.2) model and knowledge bases that store tens of thousands parameterized models and knowledge files, 2.3) platforms for multidisciplinary collaborative simulation and product data management, etc.; and 3) thousands of different kinds of CMfg capability supporting various activities in the whole lifecycle of manufacturing (e.g., design and optimization 
of multidisciplinary products, parts supply, and production process). Hierarchical gateways (i.e. management middleware) [30] were developed and used to manage the distributed manufacturing resources and capabilities before they were integrated into the aforementioned cloud with powerful computing and storage capability.

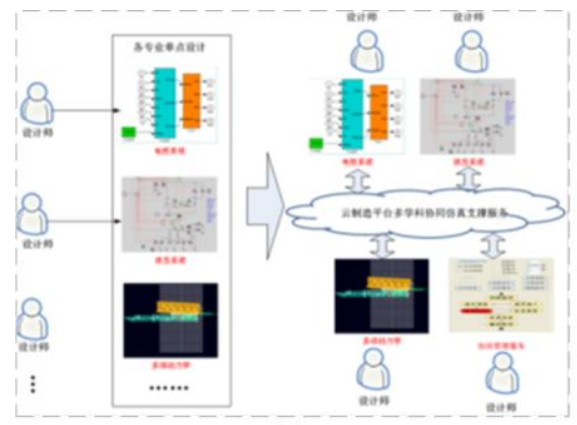

(a)

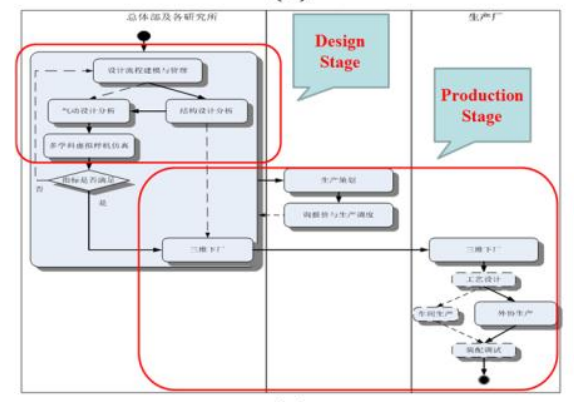

(c)

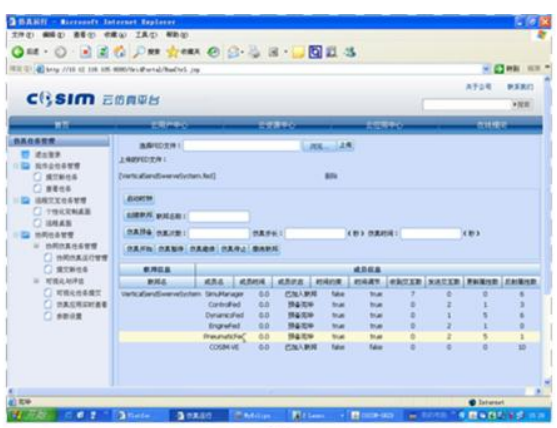

(b)

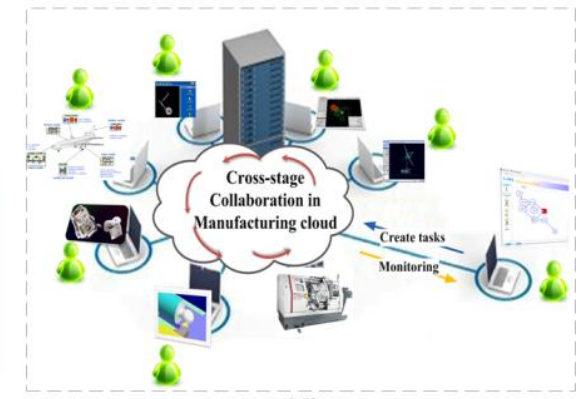

(d)

Figure 5. Single-stage and cross-stage collaborations in manufacturing cloud

The CMfg enabled open collaborative model (Fig. 1) has been adopted and partly put into practice. Those various CMfg services in the manufacturing cloud can be accessed by users on-demand. The interactions inside a single stage (e.g., collaboration between multi-disciplinary engineers in the design stage of complex products, Fig. 5 (a) (b)) or across multiple stages (e.g., engineers from design stage and production stage, Fig. 5 (c) (d)) are enabled to facilitate the collaborations, in order to improve efficiency and reduce time to market. As for the "open problem solving environment", several researchers have demonstrated the advantages of SN enabled design and manufacturing. For details, please see [4] [5] [6] [18]. Overall, the on-demand interactions between social talents beyond the organizational borders across the full lifecycle of CPPs, with the help of 
real-time information from IoT facilities, can bring great power to improve CPPs design and manufacturing.

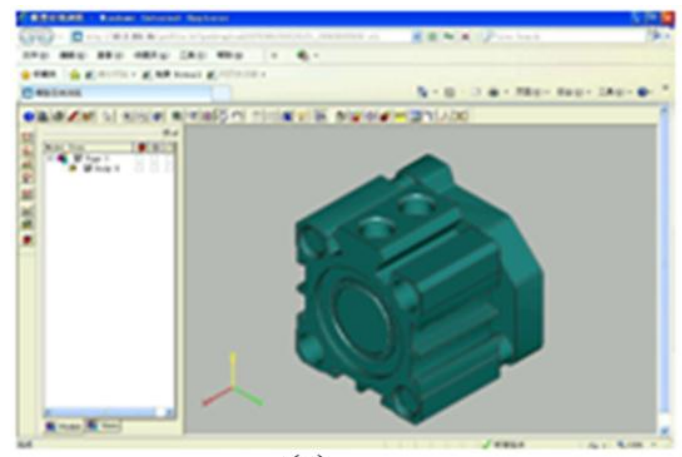

(a)

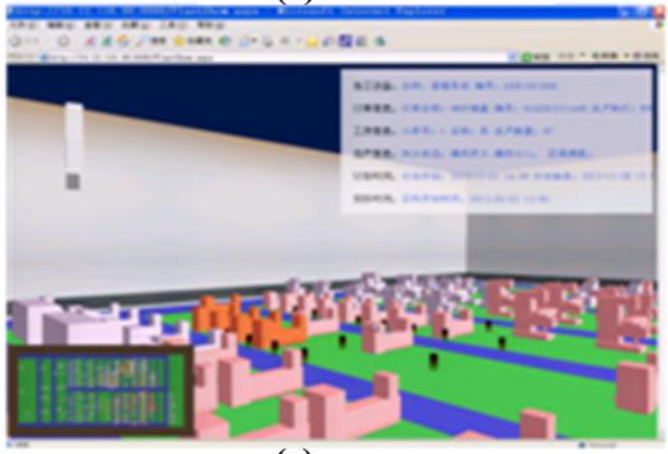

(c)

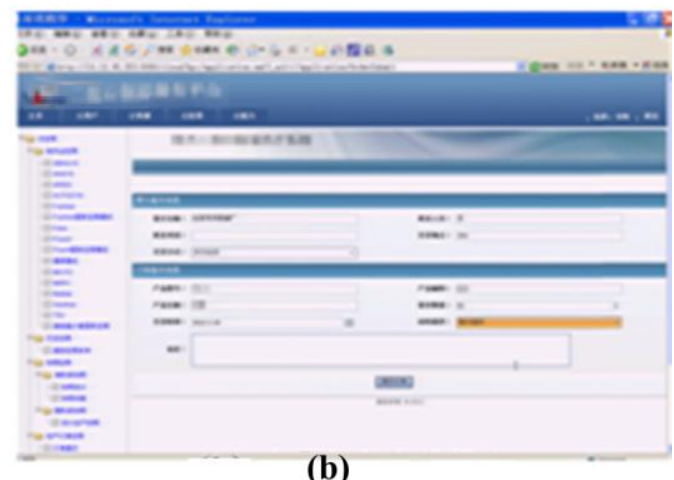

(b)

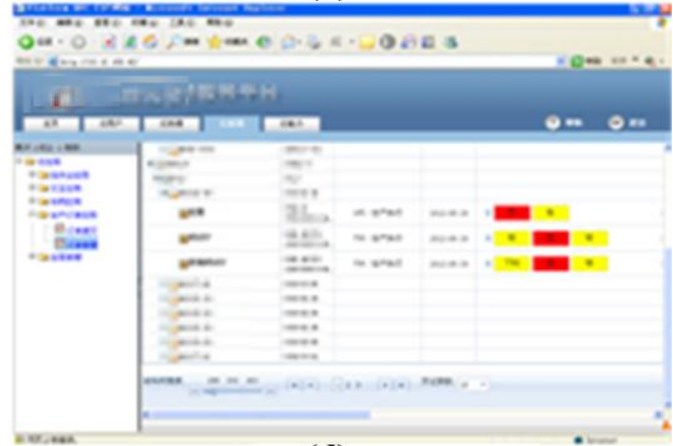

(d)

Figure 6. Prototype of the IoT-enabled workshop

In the IoT-enabled manufacturing cloud, we also implemented an IoT-enabled workshop (demonstrative), according to the design in Section 5. Collaborative design of processes for parts among participants is enabled in the cloud through the terminal devices and Internet (Fig. 6(a)). Orders can be placed remotely through web portal (Fig. 6(b)). Tens of machine tools (are numerical, or digitalized by embedment of software agents) have been integrated into the manufacturing cloud, monitored and shared in the virtual resource pool. Their static parameter (e.g., type, unique ID, etc.) and real-time information (about orders, processes, production, scheduled processing time, etc.) can be acquired and stored in the cloud. Three-Dimensional visualization and virtual walkthroughs in the workshop can be supported as shown in Fig. 6(c). RFID devices are used to track the progress of processing and assembly of parts (where tags are attached) for customer orders (Fig. 6(d)). 


\subsection{Discussions}

The complete and perfect implementation of our proposed model, architecture and "RFID-Enabled Customized/Personalized Production Based on Multi-agents" and the demonstration of their advantages still need much more effort and time. However, we can find that the following advantages are obvious and significantly beneficial.

- Customer centricity: users are deeply involved in the design and manufacturing of CPPs; support users to make the "last-minute changes to production" of customer-ordered products.

- Big Data based intelligence: Cloud based big data processing technology provides valuable knowledge from the manufacturing big data, and help all the participants to make wise decisions.

- Collective intelligence: social talents can be dynamically organized through social network and manufacturing clouds, so that they can collectively contribute to the research, development and manufacture of CPPs.

- Lifecycle traceability of CPPs: With the help of information systems, the design, production, assembly, usage and recycling information about the CPP can be traced conveniently through unique ID carried by RFID tags.

- VR/AR enabled real-time visibility and operation: use VR/AR technology to vividly display the facility status and activities in the workshop (facilitate decision making), and operate the facilities/workshop via VR/AR equipment remotely

- Flexibility: support plug \& play of new production facilities, manufacturing of CPPs and dynamic production reconfiguration.

- First time right [16]: monitor the production line to timely avoid the breakdown of machines, and control the product quality through vast deployment of sensors 
- Energy efficiency: use self-organizing networks to fast transmit the information with efficiency and leverage energy-awareness of production activities on different machines to facilitate energy saving.

- Robustness: use the self-organizing technologies to improve network reliability; when the central node and all the paths to it break down, distributed and collaborative scheduling approaches are enabled to guarantee robust production.

- Material efficiency: support the automatic planning of (residual) material reuse (e.g., of failed parts) according to the various demand of CPPs (instead of the standardized demand for materials).

To fully achieve the above advantages, our future work includes the design of optimal routing models and intelligent algorithms for the RFID-enabled customized production and the real implementation of the whole system and associated applications.

\section{Concluding Remarks}

CMfg has become a hot research spot that is pushing the reshape of the manufacturing industry. The continuous advances on IoT, SN and virtual/augmented reality \& simulation all lead to the enhanced or whole new manufacturing services in CMfg. CPPs, the latest important demand from customers also push the manufacturer to embrace those advanced technologies. However, little effort has been made to tap into vast talents and those advanced technologies to help the design and manufacturing of

CPPs. To address this issue, the paper makes the following contributions:

1) A SN-based model of full CPP lifecycle

We proposed a SN-based model for the CPP lifecycle under IoT-enabled CMfg environment. The model enhances current concurrent engineering practices (mainly design for "X") by conceiving a full interaction mode (enabled by IoT) which can bring timely information sharing in any phases to any other stages and responsive 
collaborations. It can also tap into open resources and capabilities powered by IoT-enabled CMfg and SN. CMfg can provide massive and diverse open resources and capabilities as on-demand services. IoT (real-time sensing and actuation and full networking) enables CMfg to close the loop from the manufacturing control to the dynamic adjustment of control plans. $\mathrm{SN}$ helps reach vaster majority and form like-minded communities to collectively contribute to the CPP.

2) A supporting architecture for open CPP design and manufacturing

To support the proposed model for the CPP lifecycle, we brought forward a framework for open product design and manufacturing. The framework supports five roles of users who conduct main manufacturing activities and collaborate with each other during different stages of the CPPs lifecycle. The underneath infrastructure includes three layers: IoT-enabled smart asset layer, which integrates hard and soft manufacturing resources and capabilities under the hybrid control paradigm of centralized governance and distributed autonomy; cloud operating system layer, which virtualizes integrated manufacturing resources and capabilities and provides them as on-demand services; and manufacturing application layer, which includes the main activities in an iterative loop of the CPPs lifecycle.

3) RFID enabled customized production

Due to the real-time information sharing and dynamic, efficient collaborations, dynamic configuration of customized production is beneficial to better meet customers' need. Thus we here propose an RFID enabled approach, which can execute processes step by step and change the processes according to the latest updates from participants. This will facilitate the better response to the changing needs of designers, manufacturer, consumers, etc. for CPPs, after the CPP design has been put into production, e.g., when some revisions are made afterwards. The supporting system uses a hybrid control 
method (combining centralized and distributed scheduling) which can greatly improve the system robustness. When the central node or all the paths to the central node breaks down in the centralized mode, the workstations can negotiate and collaborate to continue the production, switching into the distributed scheduling mode. The distributed scheduling can re-plan the processes responding to frequent or more dramatic topological changes of manufacturing systems and highly dynamic production activities.

We have presented some of our efforts in this paper. However, there still exists the gap to fully fulfill the potential of IoT-enabled CMfg. Researchers should try to employ IoT and CMfg to improve the collaboration efficiency in five dimensions as indicated in 4.2. With substantial support from IoT and CMfg, information can flow to anywhere it is needed, while services can be offered to anyone who needed them in a timely and efficient way. The proper visualization of assembly processes incorporating real-time IoT data to facilitate geo-distributed collaborations needs research efforts to make it reflect the instant situations. A more advanced paradigm is virtual manufacturing of the CPPs with the help of virtual/augmented reality and simulation to allow users to concurrently carry out different tasks in the full CPP lifecycle (for efficient cross-stage collaboration) [17].

SN can push the collaboration boundaries to arbitrarily large scale in the world, embracing the open talents outside. A challenging thing is how to choose the scope and scale of dynamic cooperation and how to control the quality of contributions. If too many people involve, not only may high quality contributions be overwhelmed in massive trivial and unimportant ones, but also the cost of time and money will increase significantly. 
Real-time workshop data powered by IoT and event driven methods can be used to generate alerts for predictive maintenance, cross-check the quality of processing, or evaluate the overall performance of each workstation using cloud storage and big data analytics. Experts can be organized using SN when there are irregular indexes. The inspection of the parts/products can be fed back to the designers and consumers for the CPP production improvement via proper virtual/augmented reality and simulation techniques.

\section{References}

[1] Koren, Y., Hu, S.J., Gu P., et al.: Open-architecture products. CIRP Annals-Manufacturing Technology. 62(2): 719-729 (2013)

[2] Li, B.H., Zhang, L., Wang, S.L., et al.: Cloud manufacturing: a new service-oriented networked manufacturing model. Computer Integrated Manufacturing Systems. 16(1):1-7 (2010)

[3] Manyika, J., Chui, M., Brown, B., et al.: Big data: The next frontier for innovation, competition, and productivity. (2011)

[4] Wu, D., Schaefer, D., Rosen, D.W.: Cloud-based design and manufacturing systems: a social network analysis. In: International Conference on Engineering Design, University of Bath, Bath (2013)

[5] Jiang, P., Ding, K., and Leng, J.: Towards a cyber-physical-social-connected and service-oriented manufacturing paradigm: Social Manufacturing. Manufacturing Letters, 7: 15-21 (2016)

[6] Tao, F., Cheng, Y., Zhang, L., et al.: Advanced manufacturing systems: socialization characteristics and trends. Journal of Intelligent Manufacturing. pp. $1-16(2015)$ 
[7] Ning, H., Liu, H., Ma, J., et al.: Cybermatics: Cyber-physical-social -thinking hyperspace based science and technology. Future Generation Computer Systems. 56: 504-522 (2015)

[8] Banerjee, P., Friedrich, R., Bash, C., et al.: Everything as a service: Powering the new information economy. Computer. 3: 36-43 (2011)

[9] Sudarsan, R., Fenves, S.J., Sriram, R.D., et al.: A product information modeling framework for product lifecycle management. Computer-aided design. 37(13): $1399-1411(2005)$

[10]Kagermann, H., Helbig, J., Hellinger, A., et al.: Recommendations for Implementing the Strategic Initiative INDUSTRIE 4.0: Securing the Future of German Manufacturing Industry; Final Report of the Industrie 4.0 Working Group. Forschungsunion (2013)

[11]Zhong, R.Y., Dai, Q.Y., Qu, T., et al.: RFID-enabled real-time manufacturing execution system for mass-customization production. Robotics and Computer-Integrated Manufacturing. 29(2): 283-292 (2013)

[12]Andreadis, G.: A collaborative framework for social media aware manufacturing. Manufacturing Letters. 3: 14-17 (2015)

[13]Guinard, D., Trifa, V., Karnouskos, S., et al.: Interacting with the soa-based internet of things: Discovery, query, selection, and on-demand provisioning of web services. IEEE Transactions on Services Computing. 3(3): 223-235 (2010)

[14]Gubbi, J., Buyya, R., Marusic, S., et al.: Internet of Things (IoT): A vision, architectural elements, and future directions. Future Generation Computer Systems. 29(7): 1645-1660 (2013)

[15]Park, J.H., Yang, L.T., Chen, J.: Research trends in cloud, cluster and grid computing. Cluster Computing, pp. 1-3 (2013) 
[16]Bi, Z., Da Xu, L., Wang, C.: Internet of things for enterprise systems of modern manufacturing. IEEE Transactions on Industrial Informatics. 10(2): 1537-1546 (2014)

[17]Shukla, C., Vazquez, M., Chen, F.F.: Virtual manufacturing: an overview. Computers \& Industrial Engineering. 31(1): 79-82 (1996)

[18]Jeong, H., Kim, Y.K., Kim, J.: An evaluation-committee recommendation system for national R\&D projects using social network analysis. Cluster Computing. pp. $1-10(2016)$

[19]Shen, W., Norrie, D.H.: Agent-based systems for intelligent manufacturing: a state-of-the-art survey. Knowledge and information systems. 1(2): 129-156 (1999)

[20]Tseng, M.M., Jiao, R.J., Wang, C.: Design for mass personalization. CIRP Annals-Manufacturing Technology. 59(1): 175-178 (2010)

[21]Yang, C., Shen, W., Wang, X.: Applications of Internet of Things in Manufacturing. In: Proceedings of the 2016 IEEE 20th International Conference on Computer Supported Cooperative Work in Design. pp. 670-675 (2016)

[22]Wang, F., Liu, S., Liu, P., et al.: Bridging physical and virtual worlds: complex event processing for RFID data streams. In: International Conference on Extending Database Technology. Springer Berlin Heidelberg. pp. 588-607 (2006)

[23]Bogliolo, A., Delpriori, S., Lattanzi, E., et al.: Self-adapting maximum flow routing for autonomous wireless sensor networks. Cluster Computing. 14(1): 1-14 (2011)

[24]WIKIPEDIA, https://en.wikipedia.org/wiki/DARPA_Robotics_Challenge

[25]Tao, F., Cheng, Y., Xu, L.D., et al.: CCIoT-CMfg: cloud computing and internet of things-based cloud manufacturing service system. IEEE Transactions on Industrial Informatics. 10(2): 1435-1442 (2014). 
[26]Liu, Y., Xu, X.: Industry 4.0 and Cloud Manufacturing: A Comparative Analysis. Journal of Manufacturing Science and Engineering. 139(3): 034701 (2017).

[27]Wu, D., Greer, M.J., Rosen, D.W., et al.: Cloud manufacturing: Strategic vision and state-of-the-art. Journal of Manufacturing Systems. 32(4): 564-579 (2013).

[28]He, W., Xu, L.: A state-of-the-art survey of cloud manufacturing. International Journal of Computer Integrated Manufacturing. 28(3): 239-250 (2015).

[29]Huang, G.Q., Zhang, Y.F., Jiang, P.Y.: RFID-based wireless manufacturing for real-time management of job shop WIP inventories. The International Journal of Advanced Manufacturing Technology. 36(7-8): 752-764 (2008).

[30]Lin, T.Y., Yang, C., Zhuang, C., et al.: Multi-centric management and optimized allocation of manufacturing resource and capability in cloud manufacturing system. Proceedings of the Institution of Mechanical Engineers, Part B: Journal of Engineering Manufacture. 0954405415624364 (2016). 